\title{
Resolution of racemic $\alpha$-cyclohexyl mandelic acid using chiral micromicroemulsion liquid membrane
}

\author{
Dushu Huang ${ }^{1 *}$, Yunhui Long ${ }^{1}$, Wei Liu ${ }^{1}$, Yong Hong ${ }^{1}$, Ming Zhong ${ }^{2}$, Shijuan \\ ${ }^{1}$ College of Science, Honghe University, Mengzi, Yunnan, China, 661199 \\ ${ }^{2}$ School of Chemistry and Chemical Engineering,Hunan Institute of Science and Technology, \\ Yueyang, Hunan, P. R. China, 414006 \\ a email: huangdushu2008@163.com
}

\begin{abstract}
Keywords: resolution; microemulsion liquid membrane; $\alpha$-cyclohexyl mandelic acid
\end{abstract}
\begin{abstract}
This paper deals with the resolution of racemic $\alpha$-cyclohexyl mandelic acid across microemulsion liquid membranes system using tartaric acid benzyl ester as chiral selector, sodium dodecyl sulfate as surfactant and mixed solvent of n-butyl alcohol and n-heptane as organic solvent. The effect of several variables such as chiral selector concentration, $\mathrm{pH}$ value in the external aqueous phase and volume ratio of the external aqueous phase to membrane phase was studied. Rapid extraction of $\alpha$-cyclohexyl mandelic acid and highest separation factor can be achieved while the chiral selector concentration is $20 \mathrm{mmol} / \mathrm{L}$, the $\mathrm{pH}$ value is 4.0 , and the volume ratio of the external aqueous phase to membrane phase is 1.5 .
\end{abstract}

\section{Introduction}

$\alpha$-cyclohexyl mandelic acid ( $\alpha$-CHMA) is an important precursor of chiral drugs such as oxybutynin [1]. Oxybutynin has good prospect because it is the leading drug for treatment of urinary incontinence. Pharmacological studies have found that taking racemic oxybutynin may cause dry mouth, fatigue, diarrhea and other side effects such as heart rate. However, the S-oxybutynin can reduce the side effects and has higher efficacy. Therefore, it is necessary to study separation method of high-resolution of $\alpha$ - cyclohexyl mandelic acid enantiomers. Diastereomeric resolution and HPLC separation method are usually used for racemic $\alpha$-CHMA resolution [2-4]. But the diastereomeric resolution method has some disadvantages such as long reaction steps, low yield and expensive resolving agents. And the HPLC separation method generally used for analysis.

Over recent years, resolution of chiral drugs using liquid membrane has been attracted wide attention [5-7]. Microemulsion liquid membranes are thermodymically stable dispersions which are clear, transparent system containing water, oil, surfactant and cosurfactant. It has some advantages such as low leakage and swell, low cost, simple to use, faster rate of separation and high efficiency. [8-10]. In the chiral microemulsion liquid membrane system, the internal water phase and external water phases are miscible, and they are immiscible with the organic membrane phase.

The present paper aims to study the enantioseparation of $\alpha$-cyclohexyl mandelic acid across microemulsion liquid membranes system using tartaric acid benzyl ester as chiral selector, sodium dodecyl sulfate as surfactant and mixed solvent of n-butyl alcohol and n-heptane as organic solvent. Several variables such as chiral selector concentration, $\mathrm{pH}$ value in the external aqueous phase, volume ratio of the internal aqueous phase to membrane phase and volume ratio of the external aqueous phase to membrane phase was also studied.

\section{Experiments}

\section{Materials.}

$\alpha$-cyclohexyl mandelic acid ( $\alpha$-CHMA) was purchased from Anhui Province, Guang Yuan Chemical Co., Deco, content greater than $98 \%$, the melting point of $163^{\circ} \mathrm{C} \sim 164^{\circ} \mathrm{C}$; D- tartaric acid benzyl ester (anhydrous) was purchased from Dongyang City of Hope Star biochemical Co., Ltd., 
$[\alpha]=+136^{\circ} \sim+139^{\circ},[\alpha]=-136^{\circ} \sim-139^{\circ}$, content $\geq 99 \%$, melting point:166 171 ${ }^{\circ} \mathrm{C}$. Other chemical reagents were analytical grade.

\section{Resolution process.}

The organic membrane phase was prepared by adding tartaric acid benzyl ester $(10 \mathrm{mM})$ to a mixture of $27 \%$ sodium dodecyl sulfate, $63 \%$ n-butyl alcohol and $10 \%$ n-heptane. Acetate buffer solution was used as internal stripping phase. Then the internal stripping phase and prepared organic membrane phase (15:85 to 30:70 volume basis) were stirred and water-in-oil (W/O) type microemulsion liquid membrane was achieved.

The external feed phase was prepared by adding $\alpha$-cyclohexyl mandelic acid enantiomers to acetate buffer. Then it was mixed with the microemulsion liquid membrane solution and the enantiomers can be extracted. At various times, $1 \mathrm{~mL}$ samples were removed and the $\mathrm{R}$ and S-enantiomer concentrations were determined using a $\mathrm{C}_{18}$ column. The HPLC used for analysis was a LC-2010A. The UV detector used a wavelength of $220 \mathrm{~nm}$. The column temperature was maintained at $25^{\circ} \mathrm{C}$.

\section{Result and Discussion}

\section{The effect of chiral selector concentration on enantioseparation process.}

The extraction of $\alpha$-cyclohexyl mandelic acid enantiomers is affected obviously by the concentration of chiral selector in the membrane phase. The effects of four concentrations of chiral selectors $(5,10,20,30 \mathrm{mM})$ are investigated. As shown in Fig. 1, the dimensionless concentration fractions of R-enantiomer decreased with time and the mass transfer process almost completed at about 30 seconds.

The effect of different concentration of chiral selectors in membrane phase on separation factor is shown in Fig. 2. It can be seen that the separation factor of this process increases with an increase in chiral selector concentration from 5 to $20 \mathrm{mmol} / \mathrm{L}$, and then decreases with an increase in chiral selector concentration from 20 to $30 \mathrm{mmol} / \mathrm{L}$. And the separation factor close to 2.0 can be achieved. Considering the effects of chiral selector concentration on enantiomers contration and separation factor, the concentration of chiral selectors of $20 \mathrm{mmol} / \mathrm{L}$ is selected as the optimal operating concentration.

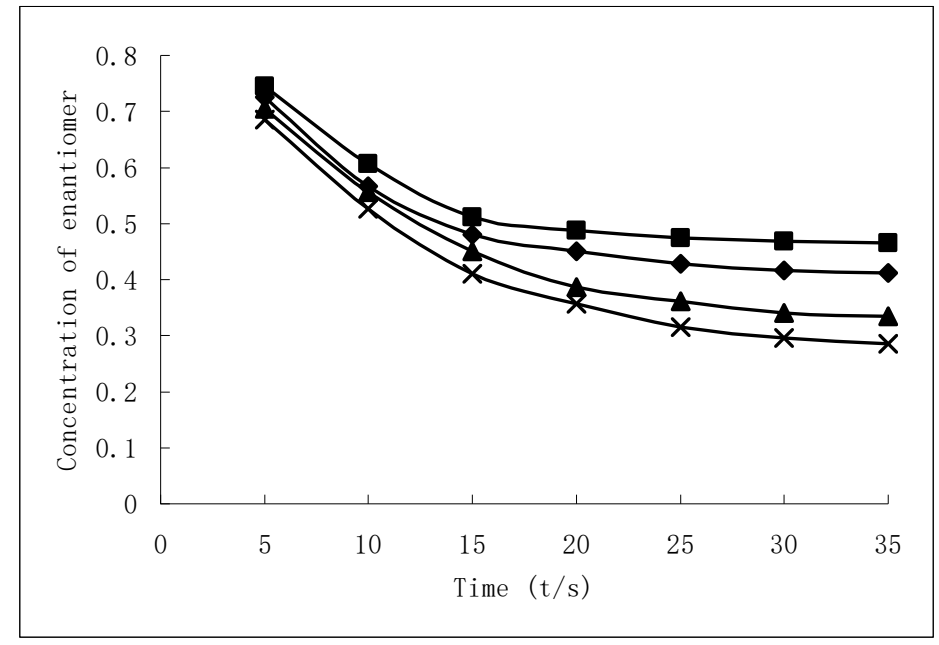

Fig. 1 The effect of concentration of chiral selectors on concentration of L-mandelic acid. $\mathbf{\square}$ : Experimental result of $5 \mathrm{mmol} / \mathrm{L}$; : Experimental result of $10 \mathrm{mmol} / \mathrm{L}$; $\mathbf{\Lambda}$ : Experimental result of $20 \mathrm{mmol} / \mathrm{L} ; \times$ : Experimental result of $30 \mathrm{mmol} / \mathrm{L}$ 


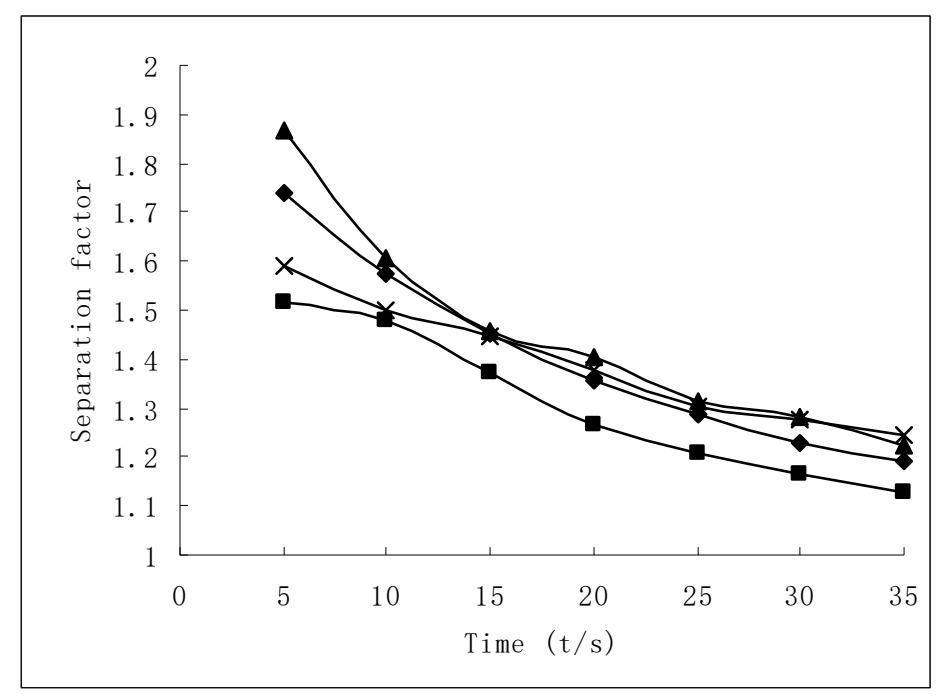

Fig. 2 The effect of concentration of chiral selectors on separation factor of chiral separation.

Experimental result of $5 \mathrm{mmol} / \mathrm{L}$; : Experimental result of $10 \mathrm{mmol} / \mathrm{L} ; \boldsymbol{\Lambda}$ : Experimental result of $20 \mathrm{mmol} / \mathrm{L}$; $\times$ : Experimental result of $30 \mathrm{mmol} / \mathrm{L}$

The effect of $\mathrm{pH}$ in external aqueous phase on enantioseparation process.

The activity of hydrogen ion in the external phase is closely connected with the ionization balance of $\alpha$-cyclohexyl mandelic acid in aqueous phase. The effects of four $\mathrm{pH}$ value (3.0, 4.0, 5.0, 6.0) are investigated. Fig. 3 shows the changes of concentration fraction of R-enantiomer with different $\mathrm{pH}$ in external aqueous phase. From the figure we can see that the extraction of $\mathrm{R}$-enantiomer increase with the $\mathrm{pH}$ value in the external aqueous phase.

The effect of $\mathrm{pH}$ in the external aqueous phase on separation factor is shown in Fig. 4. It can be seen that the initial separation factor is the highest one during the run, and it declines rapidly in the first few seconds, then it approaches to 1 as time going on. The separation factor is relatively higher when the $\mathrm{pH}$ value is 3.0 or 4.0. It is because that an increase of ionization of $\alpha$-cyclohexyl mandelic acid can be achieved with the increase of $\mathrm{pH}$ value, resulting in decrease of the number of $\alpha$-cyclohexyl mandelic acid molecules. Considering the effects of $\mathrm{pH}$ on enantiomers contration and separation factor, the $\mathrm{pH}$ value of 4.0 is selected as the optimal operating concentration.

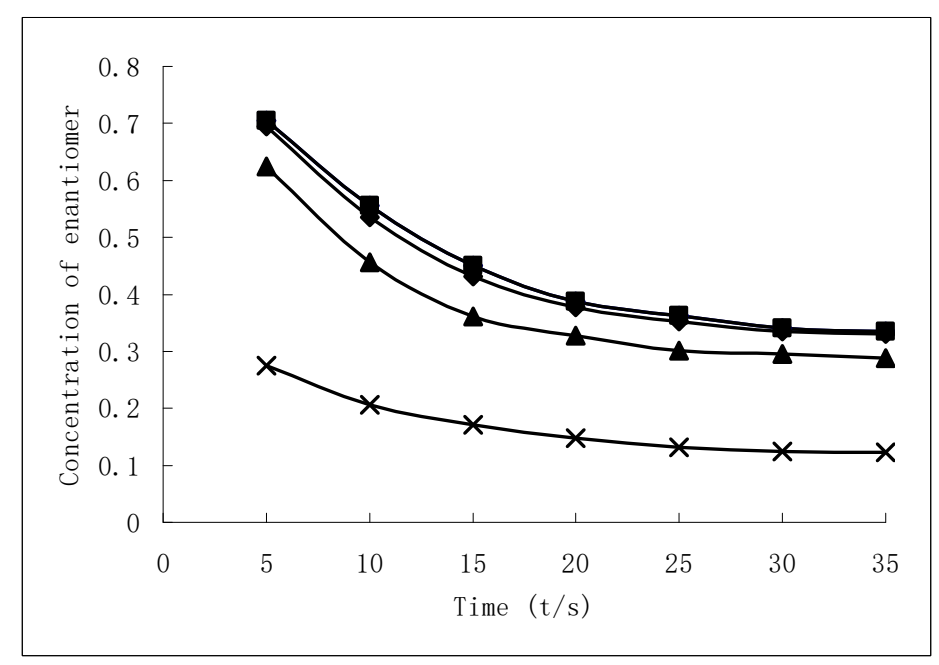

Fig. 3 The effect of $\mathrm{pH}$ of external phase on L-enantiomers concentration. $\mathbf{m}$ : Experimental result of $\mathrm{pH} 3.0$; : Experimental result of $\mathrm{pH} 4.0 ; \mathbf{\Delta}$ : Experimental result of $\mathrm{pH} 5.0 ; \times$ : Experimental result of $\mathrm{pH} 6.0$ 


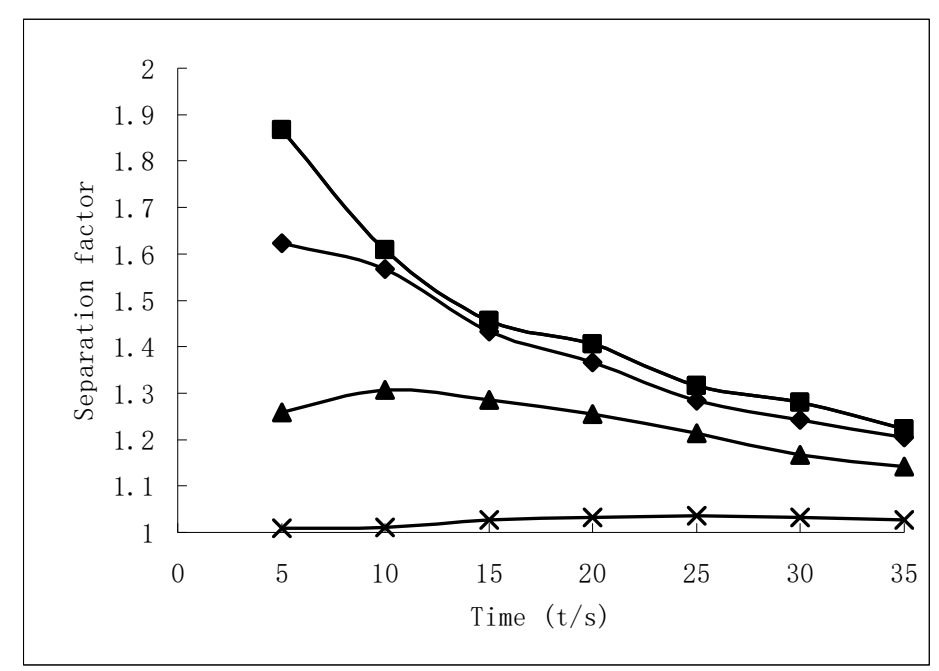

Fig. 4 The effect of $\mathrm{pH}$ of external phase on separation factor of chiral separation. $\mathbf{m}$ : Experimental result of $\mathrm{pH}$ $3.0 ;$ : Experimental result of $\mathrm{pH} 4.0 ; \mathbf{\Delta}$ : Experimental result of $\mathrm{pH} 5.0 ; \times$ : Experimental result of $\mathrm{pH} 6.0$

The effect of the volume ratio of the external aqueous phase to membrane phase on enantioseparation process.

Fig. 5 shows the changes of concentration fraction of R-enantiomer with different volume ratio of the external phase to membrane phase. The effects of four volume ratio $(1.0,1.5,2.0,2.5,3.0)$ are investigated. The extraction of R-enantiomer decreases with an increase in volume ratio of the external phase to membrane phase.

The effect of volume ratio of the external phase to membrane phase on separation factor is displayed in Fig. 6. It can be seen that the separation factor of this process increases with an increase in volume ratio of the external phase to membrane phase from 1.0 to 1.5 and from 2.0 to 3.0 , and then decreases with an increase in volume ratio from 1.5 to 2.0 at the beginning of this separation process. Considering the above effects of volume ratio on enantiomers contration and separation factor, the volume ratio of the external phase to membrane phase of 1.5 is selected as the optimal operating concentration.

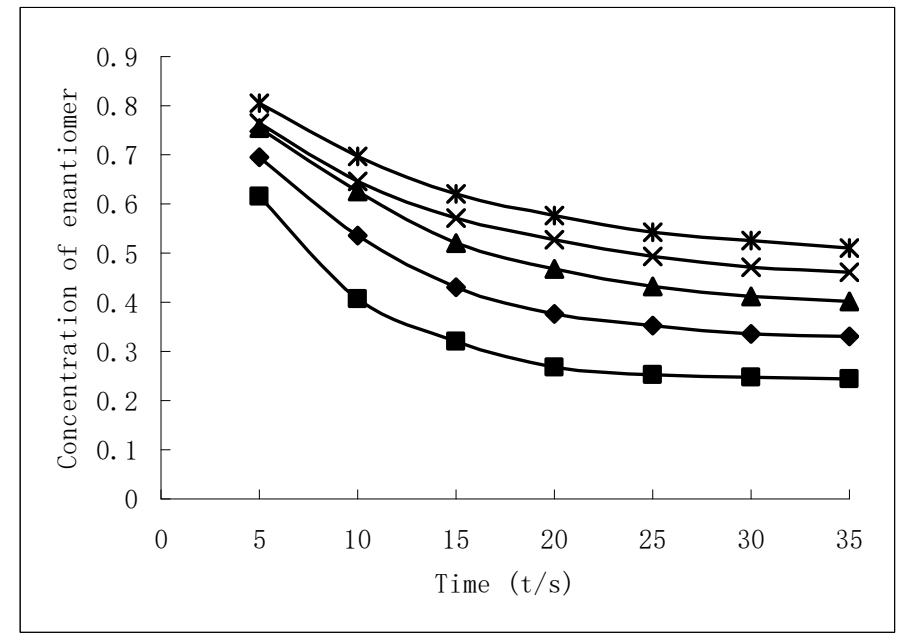

Fig. 5 The effect of volume ratio of the external aqueous phase to membrane phase on L-enantiomers concentration. $\quad \mathbf{m}$ : Experimental result of 1.0; $\$$ : Experimental result of 1.5; $\mathbf{\Delta}$ : Experimental result of 2.0; $\times$ : Experimental result of 2.5; *: Experimental result of 3.0 


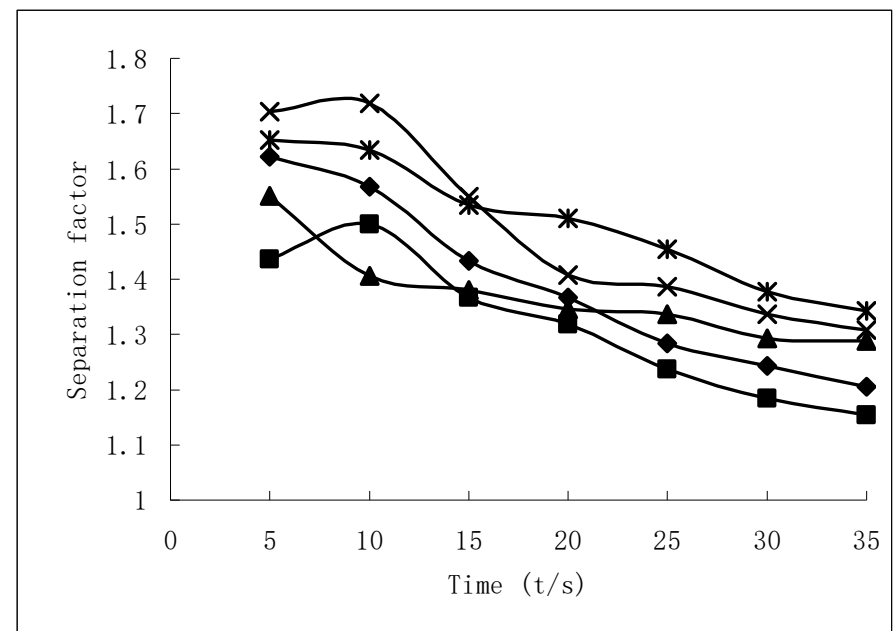

Fig. 6 The effect of volume ratio of the external phase tomembrane phase on separation factor of chiral separation. $\mathbf{\square}$ : Experimental result of $1.0 ; \bullet$ : Experimental result of $1.5 ; \boldsymbol{\Delta}:$ Experimental result of $2.0 ; ; \times$ : Experimental result of $2.5 ; *$ : Experimental result of 3.0

\section{Conclusion}

Resolution of racemic $\alpha$-cyclohexyl mandelic acid across microemulsion liquid membranes system using tartaric acid benzyl ester as chiral selector, sodium dodecyl sulfate as surfactant and mixed solvent of n-butyl alcohol and n-heptane as organic solvent was performed.

The effect of several variables such as chiral selector concentration, $\mathrm{pH}$ value in the external aqueous phase and volume ratio of the external aqueous phase to microemulsion phase was studied. Rapid extraction of $\alpha$-cyclohexyl mandelic acid and highest separation factor can be achieved while the chiral selector concentration is $20 \mathrm{mmol} / \mathrm{L}$, the $\mathrm{pH}$ value is 4.0 , and the volume ratio of the external aqueous phase to membrane phase is 1.5 .

\section{Acknowledgement}

This work was supported by the National Natural Science Foundation of China (No.21366011, $61361002,21276071,21461007)$ and the open fund of master pilot construction of chemical subject (No.HXZ1302).

\section{References}

[1] Senanayake C H, Grover P T. Stereoselective process for alkylphenylglycolic acids[P]. US: 6180823, 2001-01-30.

[2] HU Shan-shan,WU Yi-zu,SHI Mei-ren. Resolution of $\alpha$-Cyclohexyl-mandelic Acid into Enantiomers by HPLC with $\beta$-Cyclodextrin as Additive in Mobile Phase. Fine Chemicals. 2004, 21(10): 731 732

[3] Roger P B, Jorge L L, Francis X M, et al. Carbonate Intermediates useful in the preparation of optically active cyclohexylphenylglycolate [P]. US: 5973182

[4] Feitsma K. G., Bosman J., Drenth B. F. H. et al. A study of the separation of enantiomers of some aromatic carboxylic acids by high-performance liquid chromatography on a $\beta$-cyclodextrin-bonded stationary phase[J]. J. Chromatogr., 1985, 335: 59 68

[5] Chulalak Naksang, Niti Sunsandee, Nopphawat Thamphiphit, Ura Pancharoen, Prakorn Ramakul \& Natchanun Leepipatpiboon. Synergistic Enantioseparation of Rac-Phenylalanine via Hollow Fiber Supported Liquid Membrane. Separation Science and Technology. 2013, 48(6): 867-876 
[6] Chulalak Naksang, Niti Sunsandee, Nopphawat Thamphiphit, Ura Pancharoen, Prakorn Ramakul \& Natchanun Leepipatpiboon. Synergistic Enantioseparation of Rac-Phenylalanine via Hollow Fiber Supported Liquid Membrane. Separation Science and Technology. 2013, 48(6): 867-876

[7] Niti Sunsandee, Natchanun Leepipatpiboon, Prakorn Ramalul. Selective enantioseparation of levocetirizine via a hollow fiber supported liquid membrane and mass transfer prediction. Korean J. Chem. Eng., 2013, 30(6): 1312-1320

[8] Smita Gupta, Mousumi Chakraborty \& Z. V. P. Murthy. Removal of Mercury by Emulsion Liquid Membranes: Studies on Emulsion Stability and Scale Up. Journal of Dispersion Science and Technology. 2013, 34(12): 1733-1741

[9] Farhad Garavand, Ashkan Madadlou. Recovery of phenolic compounds from effluents by a microemulsion liquid membrane (MLM) extractor. Colloids and Surfaces A: Physicochemical and Engineering Aspects. 2014, 443(20): 303-310

[10]Maqsood Ahmad Malik, Mohammad Younus Wani, Mohd Ali Hashim. Microemulsion method: A novel route to synthesize organic and inorganic nanomaterials: 1st Nano Update. Arabian Journal of Chemistry. 5(4): 397-417 